\title{
Efectos de la Expansión Rápida del Maxilar sobre el Flujo Aéreo Nasal en Pacientes entre 6 y 14 Años de Edad, con Compresión Maxilar, Medido a Través de un Flujómetro Nasal Portátil
}

\author{
Effects of Rapid Maxillary Expansion on Nasal Airflow of Patients Between 6 and 14 \\ Years of Age, with Maxillary Compression, Measured with a Portable Nasal Flowmeter
}

Juan Luis Avilés Galaz'; Pamela Odet Lincovil Nancoํㄹ \& Alex Vásquez Huerta²

AVILÉS, G. J. L.; LINCOVIL, N. P. O. \& VÁSQUEZ, H. A. Efectos de la expansión rápida del maxilar sobre el flujo aéreo nasal en pacientes entre 6 y 14 años de edad, con compresión maxilar, medido a través de un flujómetro nasal portátil. Int. J. Odontostomat., 14(3):380-386, 2020.

RESUMEN: El objetivo de nuestro estudio de tipo longitudinal prospectivo simple de medidas repetidas fue determinar la variación del flujo aéreo nasal medido con un flujómetro nasal portátil, en niños entre 6 y 14 años de edad con compresión maxilar, después de la expansión rápida del maxilar (ERM). El trabajo constó de 16 niños diagnosticados con compresión maxilar y a quienes se les indicó una disyunción maxilar rápida. Los valores de la cantidad de expansión fueron registrados y la medición del flujo inspiratorio nasal máximo (FINM) se realizó antes de la ERM (T1), inmediatamente después (T2) y al cabo de 3 meses del período de retención (T3), manteniendo las mismas condiciones iniciales. El valor máximo y el promedio de las mediciones del FINM en T2 fueron significativamente mayores que en T1 ( $p$-valor, 0,0056 ) y ( $p$-valor 0,0062$)$ respectivamente, mientras que entre T2 y T3 no existieron tales diferencias ( $p$-valor: 0,3021$)$ y ( $p$-valor: 0,3315 ) respectivamente. Existe un aumento significativo en los valores del FINM inmediatamente después de la expansión rápida del maxilar que se mantienen en un período de tiempo de 3 meses.

PALABRAS CLAVE: Expansión rápida del maxilar, flujo inspiratorio nasal máximo, compresión maxilar.

INTRODUCCIÓN

La compresión maxilar es considerada una ADM intermaxilar en sentido transversal, ya que afecta la relación transversal entre el maxilar superior con el inferior. Se puede presentar en boca desde temprana edad, es de origen multifactorial y se establece de diversas maneras (Asensi, 1988). En la actualidad, existen técnicas ortodóncicas aplicadas a pacientes que presentan maloclusiones transversales, una de ellas es la expansión rápida del maxilar (ERM), procedimiento ortopédico que consiste en la disyunción palatina, incrementando de esta manera la dimensión transversal maxilar. Desde 1860, la literatura ortodóncica y del área de la rinología, reveló controversias en relación con la disyunción palatina y los cambios dimensionales no sólo a nivel de la arcada dentaria superior, sino que también a nivel de la cavidad nasal. En base a esta evidencia, el Dr. Hass en 1961 realizó una publicación en donde demostró según lo observado en ensayos clínicos y en estudios sobre animales, que cambios en la dimensión transversal del maxilar superior a través de la ERM con el uso de disyuntores maxilares, provoca también un aumento en el ancho de la cavidad nasal (Haas, 1961). Estudios recientes utilizando distintos métodos de medición, han logrado también confirmar dicha asociación (Cappellette et al., 2017).

\footnotetext{
${ }^{1}$ Programa de Especialización en Ortodoncia y Ortopedia Dentomáxilofacial, Facultad de Odontología, Universidad de Valparaíso, Chile.

${ }^{2}$ Cirujano Dentista, Ortodoncista, Profesor Titular del Programa de Especialización en Ortodoncia y Ortopedia Dentomáxilofacial, Facultad de Odontología, Universidad de Valparaíso, Chile.
} 
AVILÉS, G. J. L.; LINCOVIL, N. P. O. \& VÁSQUEZ, H. A. Efectos de la expansión rápida del maxilar sobre el flujo aéreo nasal en pacientes entre 6 y 14 años de edad, con compresión maxilar, medido a través de un flujómetro nasal portátil. Int. J. Odontostomat., 14(3):380-386, 2020.

La relación entre la ERM y el aumento en la dimensión transversal nasal podría suponer una reducción en la resistencia de las vías respiratorias nasales al paso del aire, lo que implicaría un beneficio a aquellos pacientes con un flujo aéreo nasal reducido, que muchas veces se traduce clínicamente en un hábito de respiración oral, entre otros problemas de relevancia biológica y social. Actualmente diversos estudios apoyan la relación existente entre estas dos variables, mientras que otros no son concluyentes, sobre todo en relación con la estabilidad de los resultados en el tiempo (Doruk et al., 2004; Matsumoto et al., 2010; Langer et al., 2011).

\section{MATERIAL Y MÉTODO}

Diseño, población y muestra: el diseño del estudio corresponde a un estudio "longitudinal prospectivo simple de medidas repetidas". La población de estudio fueron aquellos pacientes ingresados a tratamiento en el Postgrado de Ortodoncia y Ortopedia Dento Máxilo Facial de la Facultad de Odontología de la Universidad de Valparaíso y aquellos ingresados a tratamiento en consultas particulares de la Región de Valparaíso durante el período de tiempo comprendido entre mayo de 2018 y marzo de 2019. La muestra se conformó por 11 pacientes de la Universidad de Valparaíso y 5 pacientes de consultas particulares. De los 16 individuos, 3 eran hombres y 13 mujeres, con edades comprendidas entre los 8 y 12,9 años. Los pacientes seleccionados fueron aquellos diagnosticados con compresión maxilar y que serían sometidos a una ERM por el odontólogo tratante, además de esto, los pacientes debían cumplir con los criterios de inclusión y exclusión y estar dispuestos participar en el estudio firmando el consentimiento informado.

Tipo y cálculo de la muestral: No probabilística, no aleatoria y por conveniencia, porque permitió seleccionar aquellos casos accesibles que aceptaron ser incluidos.

Criterios de inclusión: niños (as) entre 6 y 14 años de edad con dentición mixta primer período de recambio, intertransicional, dentición mixta segundo período de recambio o dentición permanente, hasta etapa CS3 de maduración cervical según índice de Baccetti, diagnóstico de compresión maxilar (con mordida vis a vis, mordida cruzada o mordida en linguoclusión unilateral o bilateral.), pacientes con necesidad de expandir en 4 o más milímetros el maxilar superior con disyuntor maxilar según indicación del odontólogo tratante.
Criterios de exclusión: Pacientes que no cumplan con los criterios de inclusión, con tratamiento ortodóncico u ortopédico al momento del ingreso, con problemas respiratorios crónicos (rinitis alérgica estacional o perenne, enfermedad pulmonar obstructiva crónica, asma, hipertensión pulmonar, enfermedades autoinmunes que afecten las vías respiratorias, etc.), pacientes fumadores, con labio o paladar hendido, con síndromes con alteraciones craneofaciales, con alteraciones neurológicas que no puedan seguir instrucciones, hipertrofia de amígdalas palatinas mayor a grado II según Grado de Obstrucción Orofaríngea L. Brodsky y pacientes con Adenoides mayor a grado II según Porcentaje de Obstrucción Nasofaríngea.

\section{Descripción y medición de las variables}

Expansión Rápida del Maxilar (ERM): variable independiente, discreta y continua. En este estudio se utilizaron disyuntores Hyrax y McNamara según necesidad terapéutica y los valores medidos fueron entre los 4 y $14 \mathrm{~mm}$ con intervalos de $0,25 \mathrm{~mm}$. Se efectuaron 4 activaciones diarias, 2 en la mañana y 2 en la noche, equivalentes en total a $1 \mathrm{~mm}$ diario. La cantidad de expansión total fue indicada por el especialista tratante y fue ejecutada por el padre y/o apoderado. Para asegurar la correcta aplicación del protocolo, se instruyó al padre y/o apoderado por medio de una demostración clínica en el paciente, además de la entrega de una pauta y calendario de activación por escrito. Se confirmó la disyunción palatina clínicamente por la presencia de un diastema interincisivo central y por medio de una radiografía oclusal. La medición objetiva y final de la expansión se realizó con un pie de metro digital según la separación final del tornillo.

Flujo Inspiratorio Nasal Máximo (FINM): variable dependiente, continua y polidicotómica. La medición de esta variable se realizó con un Flujómetro nasal portátil (In-Check Nasal por Clement Clark International $®$ ) y se medió el FINM en L/min. La medición del flujo aéreo nasal fue llevada a cabo por los investigadores con una técnica estandarizada. Un investigador realizó todas las indicaciones a los pacientes y registró los datos, mientras que el otro sólo manipuló el instrumento de medición. El paciente debió estar sentado en posición vertical con el plano de Frankfurt paralelo al piso y con los labios en contacto. Se realizó el procedimiento hasta obtener 3 valores, mediciones que fueron consignadas para los análisis estadísticos. La primera medición del flujo aéreo nasal se realizó a todos los pacientes antes del tratamiento de ERM (T1). La se- 
AVILÉS, G. J. L.; LINCOVIL, N. P. O. \& VÁSQUEZ, H. A. Efectos de la expansión rápida del maxilar sobre el flujo aéreo nasal en pacientes entre 6 y 14 años de edad, con compresión maxilar, medido a través de un flujómetro nasal portátil. Int. J. Odontostomat., 14(3):380-386, 2020.

gunda medición se efectuó inmediatamente después de la ERM (T2). Finalmente se volvió a medir el flujo aéreo nasal al tercer mes, antes de retirar el disyuntor maxilar y después de obtener la radiografía oclusal de control que mostrara la indemnidad del aparato, es decir, que se mantuvieran las mismas condiciones iniciales y se confirmara la osificación de la sutura palatina (T3).

Análisis estadístico: El análisis estadístico se realizó por estadístico experto de la Facultad de Odontología de la Universidad de Valparaíso ajeno a la investigación. Para el análisis de resultados se utilizó estadística descriptiva de tendencia central y de dispersión para las variables cuantitativas, además de proporciones para cuantificar la magnitud de diferencias entre los distintos momentos. Dado el tamaño de la muestra, para el análisis inferencial se optó por alternativas no paramétricas, usándose la prueba de rangos de Wilcoxon para comparar dos grupos, el test de Dunn cuando la variable cualitativa tenía más de dos categorías y la prueba de correlación de Spearman para la magnitud y dirección de las mismas. Todos los análisis se realizaron con el software STATA 15.0 y se consideraron significativos $p$-valores menores a 0.05 .

\section{RESULTADOS}

La muestra estuvo conformada por 16 sujetos, de los cuales $3(18,75 \%)$ fueron de sexo masculino y $13(81,25 \%)$ femenino. Se apreció que la edad presentó una unidad de diferencia, pero no constituye diferencias significativas ( $p$-valor: 0,3126 ) debido a la variabilidad baja que presentaron dentro de cada grupo.

Ahora bien, dado que el FINM se tomó en tres intentos dentro de cada una de las sesiones, se optó por tomar el promedio de dichos intentos para obtener el valor de referencia. Se observó que inmediatamente después, entre T1 y T2 se produjeron diferencias significativas ( $p$-valor: 0,0062 ), pero entre T2 y T3 esto no ocurrió ( $p$-valor: 0,3315$)$. Ambos resultados se ven tanto en el promedio como en la mediana, los que quedan de manifiesto en la Tabla I.

Tabla I. Estadística descriptiva del promedio de FINM en los distintos tiempos medidos.

\begin{tabular}{lcccc}
\hline & Promedio & D.E. & Mediana & CV \\
\hline T1 & 103,54 & 20,77 & 98,83 & 0,20 \\
T2 & 124,31 & 22,70 & 118,33 & 0,18 \\
T3 & 120,94 & 23,07 & 114,17 & 0,19 \\
Total & 116,26 & 23,59 & 111,33 & 0,20 \\
\hline
\end{tabular}

Por otro lado, si la medición se realiza con el valor máximo de los tres intentos, los resultados son similares a los de la Tabla anterior, donde se producen cambios inmediatos entre T1 y T2 (p-valor: 0,0056), pero entre T2 y T3 no se observan diferencias significativas ( $p$-valor: 0,3021) (Tabla II).

Tabla II. Estadística descriptiva de los valores máximos del FINM en los distintos tiempos medidos.

\begin{tabular}{lcccc}
\hline & Promedio & D.E. & Mediana & CV \\
\hline T1 & 105,56 & 20,40 & 100,00 & 0,19 \\
T2 & 126,19 & 22,87 & 120,00 & 0,18 \\
T3 & 122,38 & 23,39 & 115,50 & 0,19 \\
Total & 118,04 & 23,59 & 113,00 & 0,20 \\
\hline
\end{tabular}

Para demostrar el proceso de modificación en el tiempo, se analizó el comportamiento de las diferencias producidas en los promedios de los intentos al medir el FINM, de acuerdo con los distintos tiempos en que se midió. Se aprecia que las diferencias entre el delta T3-T1 y T2-T1, no presenta diferencias significativas ( $p$-valor: 0,2659$)$, mientras que en las dos comparaciones sí existen, es decir, los mayores cambios se producen en la segunda evaluación, quedando ahora claro que la diferencia entre la T3-T2 es mas bien despreciable. Estos hallazgos se presentan en la Tabla III.

Tabla III. Estadística descriptiva de las diferencias producidas en los diferentes tiempos de acuerdo con el promedio de intentos.

\begin{tabular}{lcrcc}
\hline & Promedio & D.E. & Mediana & CV \\
\hline T3-T1 & 16,81 & 15,57 & 12,50 & 0,93 \\
T2-T1 & 20,63 & 14,56 & 17,50 & $0 ., 71$ \\
T3-T2 & $-3,81$ & 3,64 & $-3,50$ & $-0,95$ \\
Total & 11,21 & 16,34 & 8,00 & 1,46 \\
\hline
\end{tabular}

El comportamiento de los promedios de las diferencias de valores máximos obtenidos se comportó de manera similar que lo anterior. Encontrándose las magnitudes de las diferencias de delta T3-T1 y T2-T1, sin diferencias significativas ( $p$-valor: 0,2071 ). Esto se aprecia en Tabla IV.

Tabla IV. Estadística descriptiva de las diferencias producidas en los diferentes tiempos de acuerdo con el valor máximo de intentos.

\begin{tabular}{ccccc}
\hline & Promedio & D.E. & Mediana & CV \\
\hline T3-T1 & 17,40 & 15,61 & 13,17 & 0,90 \\
T2-T1 & 20,77 & 15,41 & 17,00 & 0,74 \\
T3-T2 & $-3,37$ & 2,92 & $-3,00$ & $-0,87$ \\
Total & 11,60 & 16,51 & 9,17 & 1,42 \\
\hline
\end{tabular}


AVILÉS, G. J. L.; LINCOVIL, N. P. O. \& VÁSQUEZ, H. A. Efectos de la expansión rápida del maxilar sobre el flujo aéreo nasal en pacientes entre 6 y 14 años de edad, con compresión maxilar, medido a través de un flujómetro nasal portátil. Int. J. Odontostomat., 14(3):380-386, 2020.

También es relevante identificar las magnitudes de las diferencias producidas de manera porcentual, con lo que podemos notar que el mayor cambio se produce en el lapso T2-T1. Es llamativo que el porcentaje se reduce en un $3 \%$ entre T3-T2. La diferencia entre estas comparaciones (T3-T1 y T2-T1) resultaron sin diferencias significativas ( $p$-valor: 0,2892). Así se nota que la magnitud global (T3-T1) y la diferencia a la segunda sesión (T2-T1) es donde se produce el mayor cambio en el FINM (Tabla V).

Tabla V. Porcentaje de cambio de los promedios del FINM de acuerdo con los distintos tiempos.

\begin{tabular}{cccc}
\hline & Promedio & D.E. & Mediana \\
\hline T3-T1 & $18 \%$ & $17 \%$ & $14 \%$ \\
T2-T1 & $21 \%$ & $17 \%$ & $17 \%$ \\
T3-T2 & $-3 \%$ & $3 \%$ & $-2 \%$ \\
Total & $12 \%$ & $17 \%$ & $8 \%$ \\
\hline
\end{tabular}

Al realizar la misma comparación, pero con el valor máximo, se observó que la diferencia entre estas comparaciones (T3-T1 y T2-T1) resultaron sin diferencias significativas ( $p$-valor: 0.2414) (Tabla VI).

Tabla VI. Porcentaje de cambio de los valores máximos del FINM de acuerdo con los distintos tiempos.

\begin{tabular}{cccc}
\hline & Promedio & D.E. & Mediana \\
\hline T3-T1 & $17 \%$ & $16 \%$ & $13 \%$ \\
T2-T1 & $21 \%$ & $16 \%$ & $15 \%$ \\
T3-T2 & $-3 \%$ & $3 \%$ & $-3 \%$ \\
Total & $11 \%$ & $17 \%$ & $8 \%$ \\
\hline
\end{tabular}

De acuerdo con los mm de expansión que se lograron, se puede notar que el promedio fue de $6,22 \pm 2,02 \mathrm{~mm}$ con un rango intercuantil de 2,63 y una mediana de 5.25. Esto nos dice que existe variabilidad en la muestra y que los resultados de $\mathrm{mm}$ no son homogéneos en los sujetos. Al comprobar también la relación existente entre el promedio o el valor máximo del FINM y la cantidad de milímetros de disyunción, se puede apreciar que no existiría ninguna correlación entre ambas, y con todos los p-valores mayores a 0,05 , por lo que no existe evidencia suficiente que nos afirme que ambas variables se presentan de manera correlacionada. Esto ocurre de igual manera si se diferencia por sexo, aunque llama la atención los altos valores de correlación en sexo masculino, la interpretación debe ser cuidadosa dado que eran sólo 3 sujetos.

\section{DISCUSIÓN}

El presente estudio mostró de manera cuantitativa, la variación del flujo aéreo nasal de los pacientes diagnosticados con compresión maxilar, que fueron sometidos a una ERM con disyuntores McNamara o Hyrax.

La cavidad oral al estar íntimamente relacionada con la cavidad nasal, hace suponer que cualquier constricción del paladar, diagnosticada clínicamente a través de una mordida vis a vis o cruzada uni o bilateral, puede llegar a tener también consecuencias a nivel nasal y específicamente sobre el flujo aéreo, que se puede manifestar a través de una mayor resistencia aérea nasal al momento de la inspiración. Trevisan et al. (2015), en el año 2015, en un estudio en que participaron 77 pacientes, donde 39 de ellos eran respiradores nasales y 38 bucales, se les realizó una evaluación de la ventilación nasal utilizando por una parte la medición del FINM con un flujómetro nasal portátil y por otro lado aplicando la "Escala de evaluación de síntomas de obstrucción nasal". La medición de la dimensión transversal del paladar se realizó utilizando modelos de yeso. En los análisis, se evidenció una mayor ventilación nasal en aquellos pacientes con una dimensión transversal maxilar normal tanto en la escala utilizada como en los valores del FINM, y además, se observó una permeabilidad nasal reducida y mayor grado de síntomas de obstrucción en aquellos individuos con una medida transversal maxilar menor; diferencias que entre los grupos resultaron ser significativas (Trevisan et al.).

Diversos estudios realizados en el último período de tiempo y utilizando distintas metodologías de medición, sugieren que la ERM provoca un aumento en la dimensión transversal nasal y con ello un aumento en el volumen de esta. En un trabajo publicado el año 2016, en donde participaron 61 niños con compresión maxilar y a quienes se evaluó los efectos de la ERM a través del análisis de telerradiografías frontales antes y después de los 3 meses de efectuado el procedimiento clínico; se detectó que el maxilar superior mostró un aumento para las medidas lineales de ancho maxilar y nasal después de la ERM, lo que representó por lo tanto, un aumento transversal significativo en términos estadísticos de la cavidad nasal (Cappellette et al.). Langer et al., en el año 2011, mostraron los mismos resultados, pero en un período de tiempo de 30 meses. Otros estudios utilizando TC - Cone Beam, llegaron a las mismas conclusiones (Izuka et al., 2015; Lotfi et al., 2018). Hallazgos similares por otros autores, utilizando también TC Cone Beam, fueron encontrados en los cambios 
AVILÉS, G. J. L.; LINCOVIL, N. P. O. \& VÁSQUEZ, H. A. Efectos de la expansión rápida del maxilar sobre el flujo aéreo nasal en pacientes entre 6 y 14 años de edad, con compresión maxilar, medido a través de un flujómetro nasal portátil. Int. J. Odontostomat., 14(3):380-386, 2020.

dimensionales de la cavidad nasal, pero no así a nivel orofaríngeo y nasofaríngeo, esto se podría deber probablemente a la dificultad de lograr una adecuada estandarización de la técnica radiográfica, en cuanto a la posición de la cabeza y/o de la lengua (Ribeiro et al., 2012; Motro et al., 2016). Finalmente, un estudio publicado en el año 2011 utilizando nasofibroscopía para evaluar los cambios anatómicos y volumétricos en la cavidad nasal como resultado de la ERM, demostró un aumento significativo en las mediciones finales cuando fueron comparadas con las iniciales (Edmilsson et al., 2011).

Nuestro estudio, cuyo objetivo fue determinar la variación del flujo aéreo nasal medido con un flujómetro nasal portátil, en niños entre 6 y 14 años de edad con compresión maxilar, después de la ERM, nos permitiría responder lo que la literatura no ha podido llegar a consenso, que es si estos cambios transversales que si bien son significativos en la mayoría de los estudios publicados a la fecha, podrían ser suficientes como para provocar también cambios en la ventilación nasal. Los resultados, en relación a las posibles diferencias en cuanto a sexo y edad, no son concluyentes debido al reducido número de individuos ( 3 hombres y 11 mujeres) y por el estrecho intervalo de edad de los participantes (8 y 12,9 años), sin embargo hay que destacar que los estudios publicados a la fecha, tampoco han mostrado diferencias significativas en relación con estas variables, y otros ni siquiera han realizado tales observaciones.

Los resultados del FINM utilizando el promedio, así como el valor máximo de las tres mediciones obtenidas tanto en T1 como en T2 y T3, mostraron que inmediatamente después, entre T1 y T2 se produjeron diferencias significativas, pero entre T2 y T3 no ocurrieron tales diferencias. Lo mismo se evidencia del análisis porcentual de las diferencias (promedios y valores máximos), es decir, inmediatamente después de la ERM se produce un aumento considerable del FINM que se logra mantener en el tiempo, en este caso, en un intervalo de tiempo de 3 meses, situación concordante con los resultados encontrados actualmente en la literatura; por ejemplo, Doruk en el año 2004, con una muestra de 22 niños con edad promedio de 12 años, realizó mediciones antes, durante e inmediatamente después de la ERM y también al cabo de 2,2 meses; correspondiente al período de retención. El autor también realizó mediciones después de 8 meses, para comprobar la estabilidad de los posibles cambios en el tiempo. La resistencia área nasal la evaluó en este caso utilizando rinometrías acústicas en los períodos de tiempo antes señalados. El estudio concluyó que después de la ERM se apreció una reducción de la resistencia aérea nasal, esta reducción según lo observado se mantuvo en el tiempo, dado que entre las mediciones realizadas después de la expansión y a los 8 meses, no existieron diferencias significativas (Doruk et al.). Posterior a este estudio, Oliveira De Felippe et al. (2008), en el año 2008, al analizar la relación entre la ERM y los cambios en la resistencia aérea nasal utilizando el mismo instrumento de medición que Doruk et al. observaron en este caso en 38 niños sometidos al procedimiento (edad promedio 13 años) que inmediatamente después de la ERM se produjo un $25 \%$ de reducción en la resistencia aérea nasal, a diferencia de Doruk et al. que fue del $35 \%$. Además de esto, se observó que, a los 40 días, 3 y 6 meses después de la intervención ortopédica (período de retención) y finalmente a los 9 y 12 meses de realizado el procedimiento no se registraron variaciones significativas en las lecturas (Oliveira De Felippe et al.). Monini et al. (2009), en el año 2009, en un estudio prospectivo y con una muestra mayor de niños $(n=65)$ y utilizando a diferencia de Doruk et al. y Oliverira, rinomanometrías anteriores, concluyó que la ERM provoca una reducción significativa de la resistencia aérea nasal que se logró mantener en este caso, durante 1 año (Monini et al.). En el año 2010, utilizando también rinomanometrías anteriores, Halicioglu et al. (2010) evaluaron en 15 niños las diferencias entre las resistencias aéreas nasales obtenidas antes, terminada la disyunción (8 días) y después de un período de retención de 6 meses. Este estudio entrega las mismas conclusiones manifestadas en el estudio de Monini et al. A diferencia de lo anterior, Matsumoto et al., en el año 2010, utilizando tanto la rinometría acústica como la rinomanometría anterior, midieron en 27 niños con compresión maxilar tratados con ERM, los cambios producidos en la resistencia aérea nasal después de la ERM inmediatamente después y después de 30 meses de iniciado el tratamiento. Acá se observó una disminución significativa de la resistencia aérea nasal con ambos instrumentos después de la ERM, pero que no se mantuvieron a los 30 meses. Los autores en este caso justifican estos hallazgos a posibles variables individuales de cada uno de los participantes del estudio que no fueron analizadas y que se pueden llegar a dar durante este período de tiempo (Matsumoto et al.). Al igual que el estudio anterior, en el año 2011 utilizando rinomanometrías anteriores en 25 pacientes respiradores orales y sometidos a una ERM, se logró observar que después de terminada la disyunción palatina, existió un aumento significativo en la reducción de la resistencia nasal, lo que se mantuvo estable a los 3 meses, no así a los 30 meses, en donde los valores tendieron a ser semejantes a los encontrados 
AVILÉS, G. J. L.; LINCOVIL, N. P. O. \& VÁSQUEZ, H. A. Efectos de la expansión rápida del maxilar sobre el flujo aéreo nasal en pacientes entre 6 y 14 años de edad, con compresión maxilar, medido a través de un flujómetro nasal portátil. Int. J. Odontostomat., 14(3):380-386, 2020.

antes del procedimiento. Los autores sugieren en este caso, que el aumento de la resistencia nasal se podría explicar por una hipertrofia de los cornetes nasales de tipo compensatoria aún con un aumento significativo en las dimensiones telerradiográficas a nivel nasofaríngeo, debido posiblemente al crecimiento craneofacial y a la regresión de las amígdalas a esta edad específica (Langer et al.). Al contrario, Compadretti et al. (2006) años antes, al analizar a 27 niños con compresión maxilar y tratados con ERM, observó que luego de 1 año de realizado el procedimiento y al compararse con un grupo control sano no tratado, disminuyó de manera significativa los valores de la resistencia aérea nasal, aunque los autores indican que al compararse por si sólo en el grupo tratado, los cambios fueron discretos.

Hasta el momento, la literatura ha revelado que en la mayoría de los estudios que han utilizado como instrumento de medición la rinometría acústica y rinomanometría. Estos exámenes suelen tener un costo elevado y de acceso limitado económica y geográficamente para algunos pacientes, y además de esto, requieren para su interpretación, especialistas del área médica entrenados, por lo que su aplicación clínica en el área de la odontología podría llegar a ser limitada. A pesar de la validez del flujómetro nasal portátil como instrumento de medición del flujo aéreo nasal, de su bajo costo, fácil manipulación e interpretación, ha sido pobremente utilizado en la investigación en ortodoncia y de manera nula como instrumento de uso habitual en la práctica odontológica/ ortodóncica diaria. En nuestro trabajo se utilizó un Flujómetro Clement Clarke debido a la confianza que genera la marca, considerando su reconocido rol en la manufactura de productos de prueba, monitoreo y tratamiento en el área respiratoria, trayectoria que va desde el año 1917.

En el año 2013, en un estudio que tenía por objetivo evaluar el grado de correlación entre las técnicas subjetivas y objetivas para evaluar obstrucción nasal en 184 individuos voluntarios, se evidenció que el grado de correlación existente entre las técnicas objetivas (rinometría anterior, rinomanometría y FINM) fue de moderado a fuerte, no así cuando son comparados con técnicas subjetivas (EVA y puntuación de síntomas) en donde la correlación fue nula (YepesNuñez et al., 2013). Ottaviano \& Fokkens (2016), en el año 2016, describieron en una revisión de la literatura, los diversos métodos que se pueden usar para medir la permeabilidad nasal, el flujo de aire y la resistencia aérea, de los cuales destacan el FINM, la rinomanometría anterior y la rinometría acústica. Se demostró que el FINM es reproducible en el tiempo y una buena opción para la medición de la permeabilidad nasal de manera objetiva con la ventaja de ser económico, simple y adecuado para mediciones en serie e incluso para uso doméstico en la población pediátrica. (Ottaviano \& Fokkens).

Por lo tanto, las mediciones objetivas de permeabilidad nasal a través de FINM, rinomanometría anterior y rinometría acústica, que si bien, evalúan diferentes aspectos de la obstrucción nasal de los estudios disponibles en la literatura, parece que estos métodos se correlacionan aproximadamente entre sí y que todos ellos pueden utilizarse alternativamente muy bien en la investigación, así como en la práctica clínica. El flujómetro nasal portátil, al ser de bajo costo, rápido, portátil y simple, debe estar disponible en todas las prácticas donde se diagnostiquen y traten las enfermedades de las vías aéreas, o en este caso, evaluar posibles cambios producto de un procedimiento ortopédico que involucre la vía aérea. Lo anterior también lo confirma un estudio randomizado realizado el año 2017, el cual concluye que tanto la rinomanometría como la medición del FINM son efectivas para medir variaciones aéreas nasales después de la ERM. En esta investigación se observó un aumento significativo en los valores de permeabilidad nasal luego de la ERM (Bazargani et al., 2018). En otro estudio aleatorizado, prospectivo y controlado realizado por Ottaviano et al. (2018), recientemente en el año 2018, al realizar entre otras mediciones tanto en un grupo control como grupo tratado con ERM, mostraron que el procedimiento ortopédico palatino parece mejorar significativamente la capacidad respiratoria de los pacientes tratados, al menos en términos del FINM una vez terminada la expansión y al cabo de 6 meses de realizada.

Finalmente, al comprobar la relación existente entre el promedio del FINM y la cantidad de milímetros de disyunción, se observó que no existe ninguna correlación entre ambas, y con todos los p-valores mayores a 0,05 , por lo que no existe evidencia suficiente que nos afirme que ambas variables se presentan de manera correlacionada. Esto se debe a que la cantidad de disyunción en términos de milímetros en la mayoría de los pacientes fue similar, lo que imposibilita crear subgrupos que permita buscar asociación entre estos y la cantidad de FINM lograda. Lo importante es que, en todos los pacientes, independientemente de la cantidad de disyunción realizada, el aumento del FINM fue significativo. 
AVILÉS, G. J. L.; LINCOVIL, N. P. O. \& VÁSQUEZ, H. A. Efectos de la expansión rápida del maxilar sobre el flujo aéreo nasal en pacientes entre 6 y 14 años de edad, con compresión maxilar, medido a través de un flujómetro nasal portátil. Int. J. Odontostomat., 14(3):380-386, 2020.

AGRADECIMIENTOS: agradecemos a todas las autoridades y docentes del Postrgrado de Ortodoncia y Ortopedia Dento máxilo Facial de la Universidad de Valparaíso, así como a los Directores de las clínicas particulares que participaron en nuestra investigación.

AVILÉS, G. J. L.; LINCOVIL, N. P. O. \& VÁSQUEZ, H. A. Effects of rapid maxillary expansion on nasal airflow of patients between 6 and 14 years of age, with maxillary compression, measured with a portable nasal flowmeter. Int. J. Odontostomat., 14(3):380$386,2020$.

ABSTRACT: The objective of our simple prospective longitudinal type study of repeated measures, was to determine the variation of nasal airflow measured with a portable nasal flow meter, in children between 6 and 14 years of age with maxillary compression, after rapid maxillary expansion (RME). The study consisted of 16 children diagnosed with maxillary compression and those who were identified with a rapid maxillary disjunction. The values of the amount of expansion were recorded and the measurement of the peak nasal inspiratory flow (PNIF) was performed before the ERM (T1), immediately after (T2) and after 3 months of retention period (T3), maintaining the same initial conditions. Results: the value maximum and average measurements of FINM in T2 were greater than in T1 ( $p$-value, 0.0056) and ( $p$-value 0.0062$)$ respectively, while between T2 and T3 there were no differences ( $p$ value: 0.3021 ) and ( $p$ value: 0.3315 ) respectively. There is a significant increase in PNIF values immediately after rapid maxillary expansion that is in a period of 3 months.

KEY WORDS: rapid maxillary expansion, peak nasal inspiratory flow, maxillary compression.

\section{REFERENCIAS BIBLIOGRÁFICAS}

Asensi, C. Maloclusiones Transversales. En: Canut, J. A. Ortodoncia Clínica. Barcelona, Salvat, 1988. pp.351-67.

Bazargani, F.; Magnuson, A. \& Ludwig, B. Effects on nasal airflow and resistance using two different RME appliances: a randomized controlled trial. Eur. J. Orthod., 40(3):281-4, 2018.

Cappellette, M. Jr.; Nagai, L. H. Y.; Gonçalves, R. M.; Yuki, A. K.; Pignatari, S. S. N. \& Fujita, R. R. Skeletal effects of RME in the transverse and vertical dimensions of the nasal cavity in mouth-breathing growing children. Dental Press J. Orthod., 22(4):61-9, 2017.

Compadretti, G. C.; Tasca, I. \& Bonetti, G. A. Nasal airway measurements in children treated by rapid maxillary expansion. Am. J. Rhinol., 20(4):385-93, 2006.

Doruk, C.; Sökücü, O.; Sezer, H. \& Canbay, E. I. Evaluation of nasal airway resistance during rapid maxillary expansion using acoustic rhinometry. Eur. J. Orthod., 26(4):397-401, 2004.

Edmilsson, P. J.; dos Santos-Pinto, A.; Gandini Júnior, L. G.; Guariza Filho, O. \& de Castro, A. B. B. A. T. Evaluation of the effect of rapid maxillary expansion on the upper airway using nasofibroscopy: case report and description of the technique. Dental Press J. Orthod., 16(1):81-9, 2011

Haas, A. J. Rapid expansion of the maxillary dental arch and nasal cavity by opening the midpalatal suture. Angle Orthod., 31(2):73-90, 1961.
Haliciog `lu, K.; Kiliç, N.; Yavuz, I’. \& Aktan, B. Effects of rapid maxillary expansion with a memory palatal split screw on the morphology of the maxillary dental arch and nasal airway resistance. Eur. J. Orthod., 32(6):716-20, 2010.

Izuka, E. N.; Feres, M. F. \& Pignatari, S. S. Immediate impact of rapid maxillary expansion on upper airway dimensions and on the quality of life of mouth breathers. Dental Press J. Orthod., 20(3):43-9, 2015.

Langer, M. R.; Itikawa, C. E.; Valera, F. C.; Matsumoto, M. A. \& AnselmoLima, W. T. Does rapid maxillary expansion increase nasopharyngeal space and improve nasal airway resistance? Int. J. Pediatr. Otorhinolaryngol., 75(1):122-5, 2011

Lotfi, V.; Ghoneima, A.; Lagravere, M.; Kula, K. \& Stewart, K. Threedimensional evaluation of airway volume changes in two expansion activation protocols. Int. Orthod., 16(1):144-57, 2018.

Matsumoto, M. A.; Itikawa, C. E.; Valera, F. C.; Faria, G. \& AnselmoLima, W. T. Long-term effects of rapid maxillary expansion on nasal area and nasal airway resistance. Am. J. Rhinol. Allergy, 24(2):1615, 2010.

Monini, S.; Malagola, C.; Villa, M. P.; Tripodi, C.; Tarentini, S.; Malagnino, I.; Marrone, V.; Lazzarino, A. I. \& Barbara, M. Rapid maxillary expansion for the treatment of nasal obstruction in children younger than 12 years. Arch. Otolaryngol. Head Neck Surg., 135(1):22-7, 2009.

Motro, M.; Schauseil, M.; Ludwig, B.; Zorkun, B.; Mainusch, S.; Ates, M.; Küçükkeles, N. \& Korbmacher-Steiner, H. Rapid-maxillaryexpansion induced rhinological effects: a retrospective multicenter study. Eur. Arch. Otorhinolaryngol., 273(3):679-87, 2016.

Oliveira De Felippe, N. L.; Da Silveira, A. C.; Viana, G.; Kusnoto, B.; Smith, B. \& Evans, C. A. Relationship between rapid maxillary expansion and nasal cavity size and airway resistance: short- and long-term effects. Am. J. Orthod. Dentofacial Orthop., 134(3):37082, 2008.

Ottaviano, G. \& Fokkens, W. J. Measurements of nasal airflow and patency: a critical review with emphasis on the use of peak nasal inspiratory flow in daily practice. Allergy, 71(2):162-74, 2016.

Ottaviano, G.; Maculan, P.; Borghetto, G.; Favero, V.; Galletti, B.; Savietto, E.; Scarpa, B.; Martini, A.; Stellini, E. De Filippis, C.; et al., Nasal function before and after rapid maxillary expansion in children: $A$ randomized, prospective, controlled study. Int. J. Pediatr. Otorhinolaryngol., 115:133-8, 2018.

Ribeiro, A. N.; de Paiva, J. B.; Rino-Neto, J.; Illipronti-Filho, E.; Trivino, T. \& Fantini, S. M. Upper airway expansion after rapid maxillary expansion evaluated with cone beam computed tomography. Angle Orthod., 82(3):458-63, 2012.

Trevisan, M. E.; Bellinaso, J. H.; Pacheco, A. de B.; Augé, L. B.; Silva, A. M. \& Corrêa, E. C. Respiratory mode, nasal patency and palatine dimensions. Codas, 27(2):201-6, 2015.

Yepes-Nuñez, J. J.; Bartra, J.; Muñoz-Cano, R.; Sánchez-López, J.; Serrano, C.; Mullol, J.; Alobid, I.; Sastre, J.; Picado, C. \& Valero, A. Assessment of nasal obstruction: correlation between subjective and objective techniques. Allergol. Immunopathol. (Madr.), 41(6):397401, 2013.

Dirección para correspondecia:

Juan Luis Avilés Galaz

Pamela Lincovil Nanco

Cirujanos-Dentistas

Residentes Programa de Especialización

en Ortodoncia y ODMF

Facultad de Odontología

Universidad de Valparaíso

Valparaíso - CHILE

Recibido : 11-12-2019

Aceptado: 19-02-2020

Email: juanluisaviles2@gmail.com 